\title{
Minilaparoscopic Cholecystectomy Versus Conventional Laparoscopic Cholecystectomy. An Endless Debate
}

\author{
Diego Coletta, MD, ${ }^{1,2}$ Federico Mascioli, MD, ${ }^{3}$ Andrea Balla, MD, ${ }^{3}$ \\ Francesco Guerra, MD, ${ }^{4}$ and Paolo Ossola, $\mathrm{MD}^{3}$
}

\begin{abstract}
Background: Our systematic review and meta-analysis examine the impact of minilaparoscopic cholecystectomy (MLC) versus conventional laparoscopic cholecystectomy (CLC). Some authors previously compared these surgical approaches without reaching any clear conclusion, since then, further trials have been performed, but an update was needed.

Materials and Methods: PubMed, EMBASE, and the CENTRAL were systematically searched for randomized controlled trials comparing MLC versus CLC up to August 2019. The outcome measures used for comparison were operative time (OT), overall morbidity, intra- and postoperative complications, conversion and reintervention rate, length of hospital stay (LOS), postoperative pain (POP), and cosmetic results. A meta-analysis of relevant studies was performed using RevMan 5.3.

Results: Fifteen studies, including 863 patients, were considered eligible to collect data and entered the metaanalysis. A total of 415 patients in the MLC group versus 448 in the CLC group were compared. No statistical difference as for overall morbidity, intra- and postoperative complications, conversion and reintervention rate, LOS, and cosmetic results were retrieved among the groups. CLC results faster and MLC shows to be the least painful. Conclusions: According to the available high-level evidence, both surgical approaches resulted substantially equivalent to perform LC, with some advantages of CLC as for OT and of MLC concerning POP. As a consequence, we can conclude that either procedure is superior or inferior to the other one; actually, we are not able to suggest the adoption of any of the two on a routine basis.
\end{abstract}

Keywords: minimally invasive surgery, minilaparoscopic, laparoscopic cholecystectomy, microlaparoscopic, needlescopic, miniport

\section{Introduction}

L ESS THAN 40 YEARS ago, the first laparoscopic cholecystectomy (LC) was performed as a minimally invasive alternative to open conventional approach. ${ }^{1-6}$ In the early 90's, minilaparoscopic cholecystectomy (MLC) was proposed with the aim to further reduce the invasiveness of laparoscopic procedures. ${ }^{7-10}$ Some reviews on this specific topic have already been published, with the last one published in 2011. ${ }^{11-13}$ Previous reviews compared studies reporting laparoscopic procedures performed either with minilaparoscopic instruments or with few trocars. ${ }^{11-13}$ The latest large published review does not give any clear conclusions on which is the best surgical approach due to the relative scarcity of specific evidence on the argument, with most data returning from analyses and few randomized studies returning nonunivocal results. ${ }^{13}$ Other trials have been performed on this comparison so truly advantages of MLC over the conventional laparoscopic cholecystectomy (CLC) is still unclear. The aim of the present systematic review with meta-analysis of the literature is to report the high-level evidence available comparing MLC and CLC, to elucidate and update the knowledge on this topic.

\footnotetext{
${ }^{1}$ Emergency Department-Emergency and Trauma Surgery Unit, Umberto I University Hospital, Sapienza University of Rome, Rome, Italy.

${ }^{2}$ Hepatopancreatobiliary Surgery, IRCCS - Regina Elena National Cancer Institute, Rome, Italy.

${ }^{3}$ Department of General Surgery, Umberto I University Hospital, Sapienza University of Rome, Rome, Italy.

${ }^{4}$ Department of General Surgery, Ospedali Riuniti Marche Nord, Pesaro, Italy.
} 


\section{Materials and Methods}

We conducted a systematic review of published articles according to Preferred Reporting Items for Systematic reviews and Meta-Analyses (PRISMA) guidelines ${ }^{14}$ to identify randomized controlled trials (RCTs) comparing MLC with CLC. PubMed/MEDLINE, EMBASE, and CENTRAL electronic databases ${ }^{15}$ were utilized with combination of the following search words: "minilaparoscopic cholecystectomy" or "needlescopic cholecystectomy" or "miniport cholecystectomy" or "microscopic cholecystectomy" or "minisite cholecystectomy" and "laparoscopic cholecystectomy." Data for meta-analysis were extrapolated from the included studies following the preestablished pattern (protocol registered on PROSPERO number CRD42020148335). Institutional review board approval and informed consent from participants are not required for this systematic review. Two authors
(D.C. and P.O.) performed an independent literature search up to February 2020. All articles dealing with the comparison between $\mathrm{mini} / \mathrm{microlaparoscopic/needlescopic} \mathrm{cholecystec-}$ tomy and CLC, performed using four trocars, were considered eligible, with the aim to even the sample of the analysis and compare both surgical procedures better. We adopted the definition of minilaparoscopic for all surgical procedures using one or more ports/instruments, $<5 \mathrm{~mm}$ in diameter, smaller than those used conventionally. Full-text articles considered for inclusion were appraised, and the relative references were hand-searched to find additional eligible articles. Potentially suitable studies were investigated and eventually included in the analysis if in English language; RCT comparing between MLC versus CLC; involving adult patients; and surgery performed in elective setting. Studies reporting emergency procedures or cholecystectomy with less than four trocars were excluded. Two additional authors
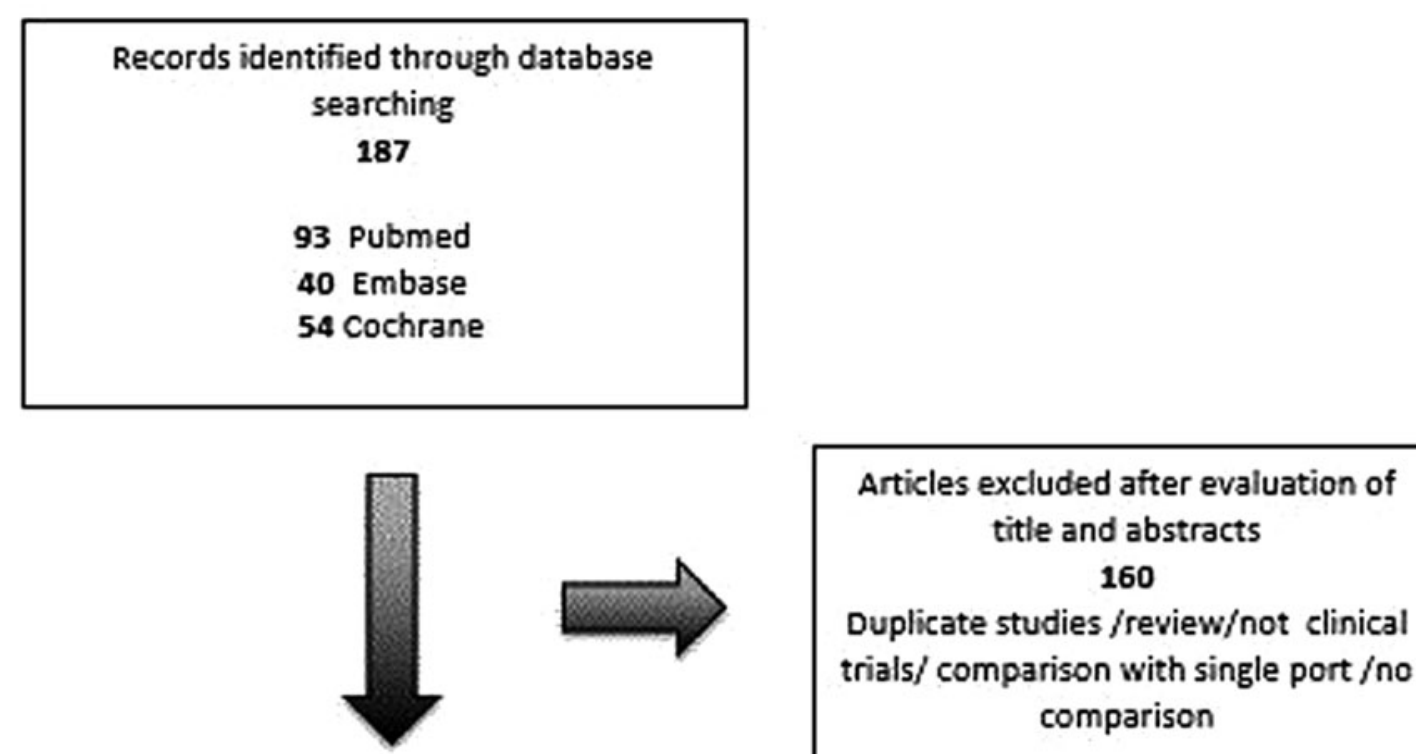

Full-text articles assessed for eligibility

27
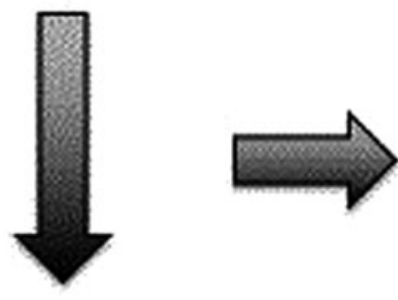

Studies included for meta-analysis 15
Records excluded on the basis of full texts

12

4 no randomization

2 emergency surgery 6 reduced trocar

FIG. 1. Flowchart of search strategy. 
(F.M. and A.B.) extrapolated data for meta-analysis from the studies that had been included. All disagreements concerning inclusion were solved by consensus, involving all authors. According to the preestablished pattern, the following data were retrieved: study design, age, body mass index (BMI), number of patients in each group, type of surgical procedure, randomization and blinding, indication for surgery, operative time (OT), conversion rate, reintervention rate, intraoperative and postoperative complications, pain scores, cosmetic outcomes evaluation, and length of hospital stay (LOS). The OT, intraoperative findings, conversion and reintervention rates, postoperative complications, LOS, postoperative pain (POP), and cosmetic results were the main outcome measures. Secondary endpoints were comparison about postoperative surgical site infections (SSI), hematoma, and incisional hernia rates. Number and size of miniport used for the MLC were also assessed.

\section{Statistical analysis}

We presented data in descriptive statistics. Meta-analysis was performed using Review Manager 5.3 (Cochrane Collaboration, Oxford, England). Estimated effect measures were calculated for event-related outcomes as odds ratio (OR). Looking over the forest plots and $I^{2}$ statistics, a statistical heterogeneity was acquired. ORs were identified and reported with confidence interval $(95 \% \mathrm{CI})$. Standard mean differences with a $95 \%$ CI by calculating random effect measures were used to analyze continuous variables presented in different scales. If RCTs reported medians and ranges instead of means and standard deviations, we converted them by using the method proposed by Hozo et al. ${ }^{16}$ The $Z$-test for overall effect and its two-sided $P$ value were assessed. The cutoff for statistical significance was set at the 0.05 probability level.

\section{Results}

\section{Studies selection and quality assessment}

The first electronic search yielded 187 records. After the evaluation of abstracts, full-texts, and references, 15 studies, including a total of 863 patients, met the inclusion criteria and entered the meta-analysis, 415 in the MLC group and 448 in the CLC group (Fig. 1 depicts our search strategy). We performed a quality analysis assessing risk of bias according the Cochrane Collaboration tool ${ }^{17}$ (Fig. 2 shows risk of bias summary), and we appreciated blinding process of the included studies. $^{18}$

\section{Characteristics of studies}

Ten of the included studies were from Europe, ${ }^{19-28} 3$ from Asia, ${ }^{29-31} 1$ from Canada, ${ }^{32}$ and 1 from the United States. ${ }^{33}$ All studies were RCTs, 14 compared MLC and CLC, and 1 considered also the single-port cholecystectomy (SPC). ${ }^{25}$ In the latter study, we excluded data relative to SP procedures. Table 1 summarizes characteristics of the included studies.

\section{Primary endpoints}

Operating time. All included studies indicated operating time in minutes. ${ }^{19-33}$ Overall, the CLC group was significantly faster than MLC. Mean OT varied between $35 \pm 14$
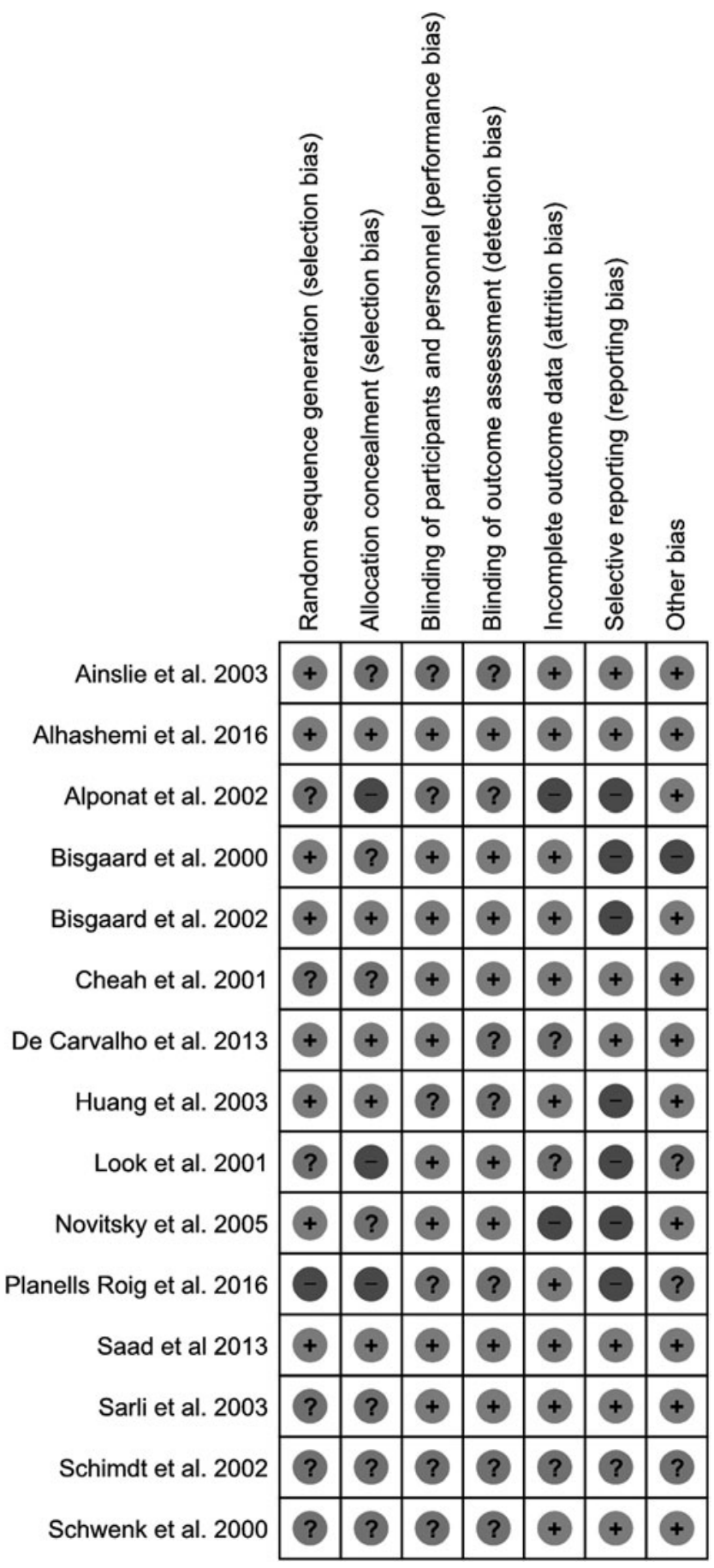

FIG. 2. Risk of bias summary.

and $75 \pm 39.8$ minutes for CLC and between $36.5 \pm 12.6$ and $92.5 \pm 31.8$ for MLC. Meta-analysis showed a significant difference in favor of CLC (mean difference [MD] 4.77, 95\% CI 3.29-6.25 $P=<.00001 ; I^{2}=57 \%$ ). (Fig. 3).

Intraoperative findings. In 9 studies, ${ }^{19,23-28,30,33}$ data on intraoperative bleeding are reported with no difference between MLC and CLC (OR 0.37, 95\% CI 0.12-1.14, $P=.08$; $I^{2}=0 \%$ ).

Gallbladder perforation occurred in 28 patients in MLC and 30 patients in CLC group. Data were available from 


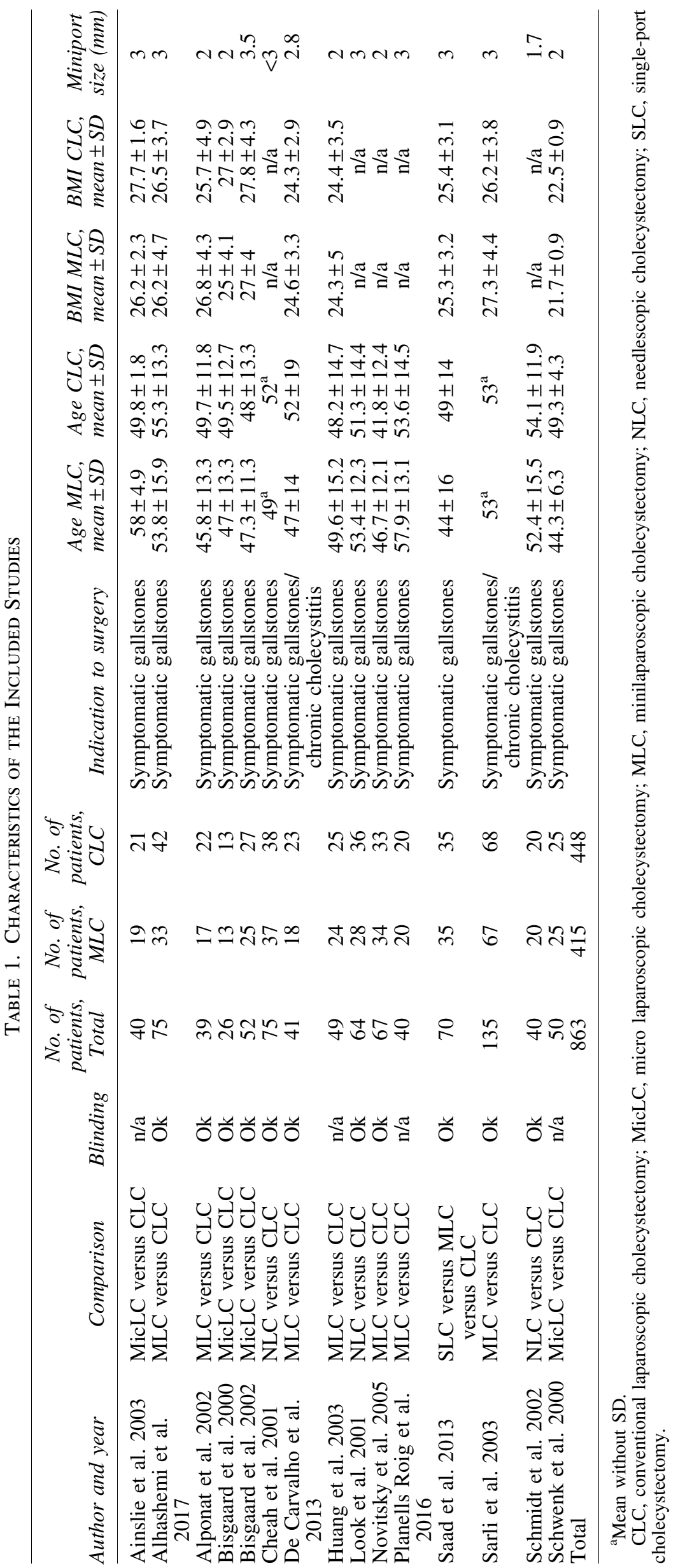




\begin{tabular}{|c|c|c|c|c|c|c|c|c|c|}
\hline \multirow[b]{2}{*}{ Study or Subgroup } & \multicolumn{3}{|c|}{ MLC } & \multicolumn{3}{|c|}{ CLC } & \multicolumn{2}{|r|}{ Mean Difference } & \multirow{2}{*}{$\begin{array}{l}\text { Mean Difference } \\
\text { IV, Fixed, } 95 \% \mathrm{Cl}\end{array}$} \\
\hline & Mean & SD & Total & Mean & SD & Total & Weight & IV, Fixed, 95\% Cl & \\
\hline Ainslie et al. 2003 & 75 & 9.3 & 19 & 64.6 & 7.2 & 21 & $8.1 \%$ & $10.40[5.21,15.59]$ & \\
\hline Alhashemi et al. 2016 & 73 & 22 & 33 & 67 & 15 & 42 & $2.8 \%$ & $6.00[-2.77,14.77]$ & \\
\hline Alponat et al. 2002 & 80.9 & 18.4 & 17 & 72.1 & 24.3 & 22 & $1.2 \%$ & $8.80[-4.60,22.20]$ & \\
\hline Bisgaard et al. 2000 & 92.5 & 31.8 & 13 & 80 & 43.3 & 13 & $0.3 \%$ & $12.50[-16.70,41.70]$ & \\
\hline Bisgaard et al. 2002 & 85 & 40 & 25 & 62 & 22.5 & 27 & $0.7 \%$ & $23.00[5.17,40.83]$ & \\
\hline Cheah et al. 2001 & 50 & 5.4 & 37 & 45 & 4.3 & 38 & $44.6 \%$ & $5.00[2.79,7.21]$ & \\
\hline De Carvalho et al. 2013 & 45 & 7 & 18 & 42 & 9 & 23 & $9.1 \%$ & $3.00[-1.90,7.90]$ & \\
\hline Huang et al. 2003 & 64.8 & 27.7 & 24 & 47.3 & 20.8 & 25 & $1.2 \%$ & $17.50[3.74,31.26]$ & \\
\hline Look et al. 2001 & 72.1 & 21.6 & 28 & 75.1 & 39.8 & 36 & $0.9 \%$ & $-3.00[-18.27,12.27]$ & \\
\hline Novitsky et al. 2005 & 50.5 & 15.4 & 34 & 54.9 & 22.4 & 33 & $2.6 \%$ & $-4.40[-13.63,4.83]$ & \\
\hline Planells Roig et al. 2016 & 36.5 & 12.6 & 20 & 46.6 & 22.7 & 20 & $1.7 \%$ & $-10.10[-21.48,1.28]$ & \\
\hline Saad et al 2013 & 47.3 & 17.7 & 35 & 35 & 14 & 35 & $3.9 \%$ & $12.30[4.82,19.78]$ & \\
\hline Sarli et al. 2003 & 50 & 37.5 & 67 & 45 & 25 & 68 & $1.9 \%$ & $5.00[-5.77,15.77]$ & \\
\hline Schimdt et al. 2002 & 51 & 25.5 & 20 & 39 & 19.5 & 20 & $1.1 \%$ & $12.00[-2.07,26.07]$ & \\
\hline Schwenk et al. 2000 & 71.8 & 6.8 & 25 & 70 & 5 & 25 & $20.0 \%$ & $1.80[-1.51,5.11]$ & \\
\hline Total $(95 \% \mathrm{Cl})$ & & & 415 & & & 448 & $100.0 \%$ & $4.77[3.29,6.25]$ & \\
\hline \multicolumn{9}{|c|}{ Heterogeneity: $\mathrm{Chi}^{2}=32.41, \mathrm{df}=14(P=0.004) ; \mathrm{I}^{2}=57 \%$} & $\begin{array}{ccc}-10 & 0 & 10 \\
\text { ours MLC } & \text { Favours }\end{array}$ \\
\hline
\end{tabular}

FIG. 3. Operative times. CI, confidence interval; CLC, conventional laparoscopic cholecystectomy; MLC, minilaparoscopic cholecystectomy; SD, standard deviation.

four articles ${ }^{23,25,26,32}$ (OR 1.09, 95\% CI 0.61-1.94P=.77; $\left.I^{2}=59 \%\right)$.

Conversions. Conversions were reported in 14 studies. ${ }^{19-24,26-33}$ Overall conversion rate was $2.3 \%$, with incidence of $2.1 \%$ and $2.9 \%$ in MLC and CLC group, respectively. Statistically significant differences between the two approach were not highlighted in the meta-analysis (OR $0.77,95 \%$ CI $0.32-1.88 P=.57 ; I^{2}=0 \%$ ). MLC to CLC conversion rate was $13.4 \%$.

Reintervention. Data on reintervention rate was reported in 13 studies. ${ }^{19,20,22-28,30-33}$ Overall, a total of 5 patients $(0.6 \%)$ underwent a reintervention, 4 in CLC group and 1 in the MLC group $(P=.28)$.

Postoperative complications. Data concerning overall morbidity were reported by 12 studies ${ }^{19,20,23-28,30-33}$ with 16 versus 22 adverse events in MLC and CLC group, respectively. Eleven studies ${ }^{19,20,23-26,28,30-33}$ analyzed data on bile duct injury (BDI). One study reported two BDIs during CLC. ${ }^{31}$

Ten studies reported bowel injury data, one of these reported one lesion during MLC. ${ }^{32}$ No significant differences comparing the groups were noted.

Hospital stay. Seven studies ${ }^{20,22,23,25-27,29,30}$ reported data on LOS: in one of them, ${ }^{20}$ all patients were discharged in the first postoperative day (POD). Statistically insignificant differences were observed between the two surgical approaches (MD $0.00,95 \% \mathrm{CI}-0.32$ to $0.32 P=.98 ; I^{2}=64 \%$ ).

Postoperative pain. Thirteen studies reported results about POP ${ }^{19,20,22-33}$ with a variety of scales and methods for pain evaluation. The most common method was the visual analog scale (VAS). ${ }^{19,20,22,24,25,27-29,31-33}$ However, in two

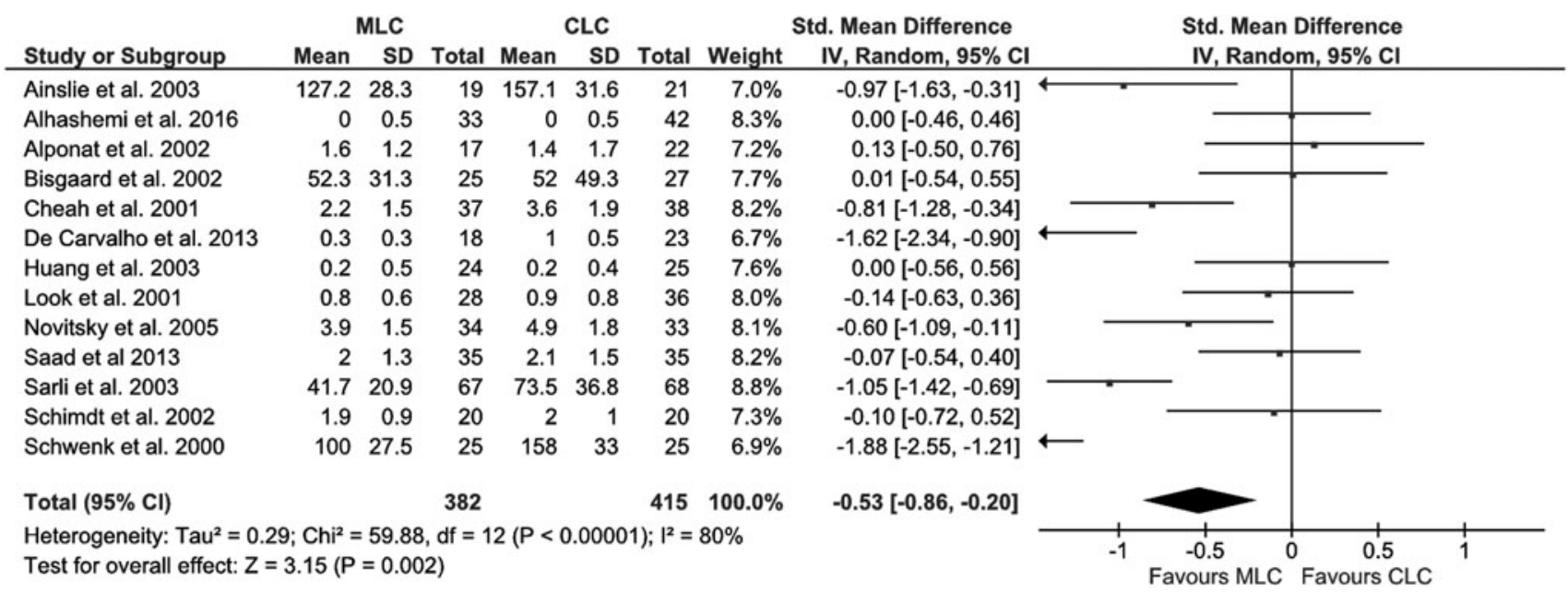

FIG. 4. POP pooled analysis. CI, confidence interval; CLC, conventional laparoscopic cholecystectomy; MLC, minilaparoscopic cholecystectomy; POP, postoperative pain; SD, standard deviation. 


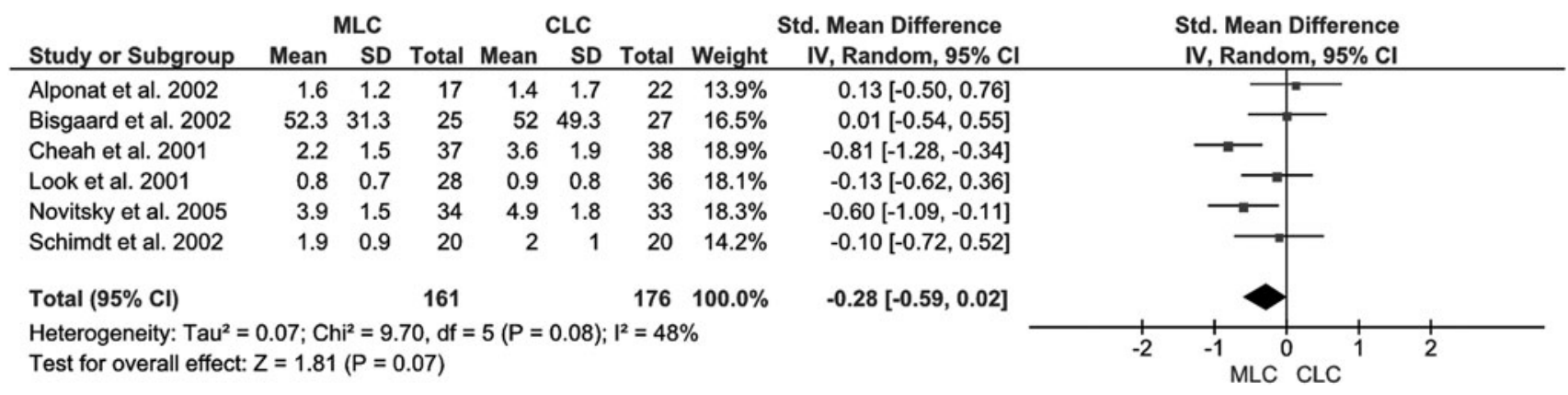

FIG. 5. Forest plot of POP in first POD analysis. CI, confidence interval; CLC, conventional laparoscopic cholecystectomy; MLC, minilaparoscopic cholecystectomy; POD, postoperative day; POP, postoperative pain; SD, standard deviation.

trials, ${ }^{26,30}$ POP value depended on the analgesic drugs consumption, in the other two, ${ }^{25,28}$ it was indicated as the cumulative VAS score until the third POD, and in one study, ${ }^{32}$ VAS was performed at 30 POD. In one case, ${ }^{23}$ POP was established with numerical rating scale (NRS) during the first POD. The pooled data analysis shows statistically significant reduced pain in MLC group (MD $0.53,95 \%$ CI -0.86 to 0.20 $\left.P=.002 ; I^{2}=80 \%\right)$. However, we performed a meta-analysis of those studies that evaluate POP by using the VAS scale only on the first POD and the data did not confirm the previous reported results ${ }^{20,22,27,29,31,33}$ (MD $-0.28,95 \% \mathrm{CI}$ -0.59 to $-0.02 P=.07 ; I^{2}=48 \%$ ) (Figs. 4 and 5 ).

Cosmetic results. Eight RCTs assessed cosmetic results utilizing a variety of scales and scores. ${ }^{20,22,23,25,28,30,32,33}$ In the analysis of the pooled data, the comparison does not reach statistically significant difference, showing high heterogeneity among the studies (MD $0.57,95 \%$ CI -0.73 to 1.88 $P=.39 ; I^{2}=97 \%$ ) (Fig. 6).

\section{Secondary endpoints}

Postoperative SSI. Twelve studies reported data on SSI with a total of 9 events, overall 2 in MLC group and 7 in the CLC group, without statistically significant differences. $^{19,20,23-28,30-33}$ (OR 0.45, 95\% CI 0.13-1.60 P=.22; $\left.I^{2}=0 \%\right)$.

Hematoma. Among the 6 studies ${ }^{19,24-26,28,33}$ that analyzed the incidence of postoperative hematoma, only Ainslie et al. ${ }^{19}$ reported 3 cases in MLC group, with statistically insignificant difference (OR 9.12, 95\% CI 0.44-189.13 $P=.15)$.

Incisional hernia. Six studies reported the incidence of umbilical postoperative hernia. ${ }^{24-28,31}$ Only one event in CLC group was reported in the series of Sarli et al. ${ }^{26}$ and no statistically significant differences are observed (OR 0.33, 95\% CI 0.01-8.33 $P=.50)$. All intraoperative and postoperative findings are summarized in Table 2.

\section{Discussion}

This meta-analysis aimed to compare two different but similar minimally invasive surgical approaches to perform cholecystectomy: the less painful MLC and the faster CLC. Some reviews about the comparison between MLC and CLC have already been published in the literature ${ }^{11-13}$; however, in the last decade, further studies have been made available, ${ }^{23-25,32,34,35}$ hence, we decided to analyze this topic, including the new evidence with the attempt to reach a clear statement. One of the previous reviews ${ }^{11}$ reported that MLC is a valid alternative to CLC in terms of POP and cosmetic results; another one ${ }^{12}$ reported some limited improvements in surgical outcomes after MLC and a high risk of conversion in CLC or open surgery and adverse events; the last one ${ }^{13}$ reported unclear conclusions about which is the best surgical approach due to the relative lack of high-quality methodologies used in the study analysis, with most data returning from few randomized studies reporting nonunivocal

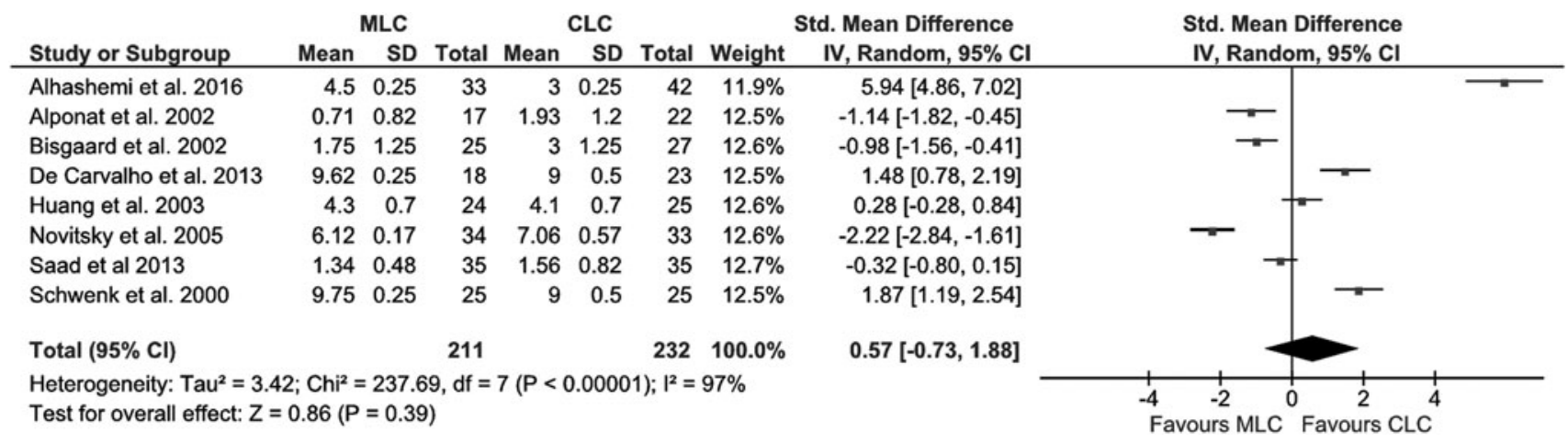

FIG. 6. Cosmetic results. CI, confidence interval; CLC, conventional laparoscopic cholecystectomy; MLC, minilaparoscopic cholecystectomy; SD, standard deviation. 
Table 2. Intraoperative and Postoperative Findings

\begin{tabular}{lccc}
\hline & $M L C$ & $C L C$ & $\mathrm{P}$ \\
\hline Operative time, median \pm SD & $61.3 \pm 15.9$ & $55.7 \pm 16.2$ & $<.00001$ \\
Bleeding, $n(\%)$ & $4(1)$ & $11(2.4)$ & .08 \\
Gallbladder perforation, $n(\%)$ & $28(6.7)$ & $30(6.7)$ & .77 \\
Overall conversions, $n(\%)$ & $8(2.1)$ & $12(2.9)$ & .57 \\
Reintervention, $n(\%)$ & $1(0.3)$ & $4(1)$ & .28 \\
Bile duct injury, $n(\%)$ & $0(0)$ & $2(0.6)$ & .37 \\
Bowel injury, $n(\%)$ & $1(0.3)$ & $0(0)$ & .41 \\
LOS, range & $0.5 \pm 0.5-3.4 \pm 0.7$ & $0 \pm 0.5-3.7 \pm 1$ & .98 \\
POP, range & $0 \pm 0.5-127.2 \pm 28.3$ & $0.9 \pm 0.5-158 \pm 33$ & .002 \\
POP 1 PoD, range & $0.8 \pm 0.7-52.3 \pm 31.3$ & $1.93 \pm 1.2-9 \pm 0.3$ & .09 \\
Cosmetic, range & $0.71 \pm 0.82-9.75 \pm 0.25$ & $7(1.9)$ & .39 \\
SSI, $n(\%)$ & $2(0.6)$ & $0(0)$ & .22 \\
Hematoma, $n(\%)$ & $3(1.5)$ & $1(0.5)$ & .15 \\
Incisional hernia, $n(\%)$ & $0(0)$ & .50 \\
\hline
\end{tabular}

The significance of bold values is $P<0.05$.

Data are reported in median with standard deviation, percentage, and range of median with standard deviation.

CLC, conventional laparoscopic cholecystectomy; LOS, length of hospital stay; MLC, minilaparoscopic cholecystectomy; PoD, postoperative day; POP, postoperative pain; SSI, surgical site infection.

results. Moreover, previous studies reported data of surgical procedures performed using a large heterogeneity of number and size of trocars. ${ }^{11-13}$ This difference is due probably to the different meaning adopted to define MLC, including the reduced trocar surgeries. Furthermore, in the previous reviews, emergency procedures were included in the analysis. ${ }^{1-13} \mathrm{In}$ the present meta-analysis, only studies reporting surgical procedures performed with four trocars and in elective settings were included, with the aim to obtain homogeneous data. For the same reasons, SP procedures were excluded, being not comparable to MLC or CLC. Results concerning OT of previous published reviews are discordant, Hosono and Osaka and Thakur et al. reported shorter OT in the case of $\mathrm{CLC}^{11,13}$ and conversely McCloy et al. reported in the case of MLC. ${ }^{12}$ Our study has shown significant reduced OT in the case of CLC group over MLC one. As expected, considering the comparisons of the two approaches, no significant difference was observed in terms of overall morbidity, intraoperative bleeding, gallbladder perforation, and bile duct and bowel injury, also when individually analyzed, as reported in previous articles. ${ }^{12,13}$ Overall, open conversion rate was $2.3 \%$, with an incidence of $2.1 \%$ and $2.9 \%$ in MLC and CLC, respectively, without showing any significant difference, in contrast with those previously published. Percentage of MLC converted in CLC has been also assessed resulting in $13.4 \%$. All considered studies and previous cited reviews analyzed conversion rate of MLC in open surgery and in CLC pooling them. According to us, these methods could result in a selection bias conducting in an obviously higher overall conversion rate for MLC group.

Reported data regarding POP are very heterogeneous $\left(I^{2} 97 \%\right)$ in terms of type of scale and time of evaluation. VAS scale was used in the majority of considered trials, varying from 1 to $30 \mathrm{POD}^{26,30}$; other RCTs adopted cumulative VAS score or NRS scales. ${ }^{19,20,22-25,27-29,31-33}$ MLC group had significant reduced pain at the pooled data analysis, but these results were not confirmed if meta-analysis includes only the studies with VAS score at 1 POD. ${ }^{20,22,27,29,31,33}$

High variability among scales used to evaluate cosmetic results was also detected, without observing any statistically significant difference between the two groups. Regarding this outcome, the previous published studies are in contrast, earlier reviews reported better cosmetic results for patient undergone MLC, while recent CLC shows superiority in comparison to MLC; in this case, the lack of standardization in cosmetic evaluation affects the results.

About LOS, no significant difference between the groups was observed in this study, but data were available from less than half of all included studies. ${ }^{20,22,23,25-27,29,30}$ Moreover, in one study, patients were discharged on first POD. ${ }^{20}$

Regarding the secondary endpoints, as for postoperative SSI, hematoma and incisional hernia were not observed as statistically significant differences in any of the considered outcomes.

The main limitation of the present study is the heterogeneity of definition of MLC adopted in the analyzed studies that could affect the data extraction and the outcome evaluations, despite our effort to homogenize the sample of the analysis. The lack of standard scales to measure POP and cosmetic outcomes could lead to wrongful conclusions in the comparison of these two similar surgical approaches. Most of the considered outcome measures, resulting from data not reported in all included studies, could affect final results, giving us a statistical illusion that eventually could not reflect reality.

\section{Conclusions}

In conclusions, according to the highest quality evidence data in literature, CLC is faster than MLC, but the second one shows to be less painful. Both surgical approaches resulted similar in overall morbidity, intra- and postoperative complications, conversion and reintervention rates, cosmetic results, and LOS. Based on the present meta-analysis, it is not possible to suggest one approach rather than the other.

\section{Acknowledgment}

We thank Anna Contrafatto for the English revision of the article.anna.contrafatto@outlook.com 


\section{Disclosure Statement}

No competing financial interests exist.

\section{Funding Information}

No grants or funding have been received for the drawing up of the present paper.

\section{References}

1. Lukichev OD, Filimonov MI, Zybin IM. A method of laparoscopic cholecystostomy. Khirurgiia (Mosk) 1983; 125-127.

2. Mühe E. The first laparoscopic cholecystectomy. Langenbecks Arch Chir 1986;369(Suppl):804.

3. McKernan JB, Saye WB. Laparoscopic general surgery. J Med Assoc Ga 1990;79:157-159.

4. Reddick EJ, Olsen DO. Laparoscopic laser cholecystectomy. A comparison with mini-lap cholecystectomy. Surg Endosc 1989;3:131-133.

5. Dubois F, Icard P, Berthelot G, Levard H. Coelioscopic cholecystectomy. Preliminary report of 36 cases. Ann Surg 1990;211:60-62.

6. Mouret P. How I developed laparoscopic cholecystectomy Ann Acad Med Singapore 1996;25:744-747.

7. Yuan RH, Lee WJ, Yu SC. Mini-laparoscopic cholecystectomy: A cosmetically better, almost scarless procedure J Laparoendosc Adv Surg Tech A 1997;7:205-211.

8. Gagner M, Garcia-Ruiz A. Technical aspects of minimally invasive abdominal surgery performed with needlescopic instruments. Surg Laparosc Endosc 1998;8: 171-179.

9. Davides D, Vezakis A, Lianyu Y, Dexter SPL, Martin IG, Larvin M, et al. Early experience with micropuncture laparoscopic cholecystectomy. Surg Endosc 1997;11:513.

10. Ngoi SS, Goh P, Kok K, Kum CK, Cheah WK. Needlescopic or minisite cholecystectomy. Surg Endosc 1999;13: 303-305.

11. Hosono S, Osaka H. Minilaparoscopic versus conventional laparoscopic cholecystectomy: A meta-analysis of randomized controlled trials. J Laparoendosc Adv Surg Tech A 2007;17:191-199.

12. McCloy R, Randall D, Schug SA, Kehlet H, Simanski $\mathrm{C}$, Bonnet $\mathrm{F}$, et al. Is smaller necessarily better? A systematic review comparing the effects of minilaparoscopic and conventional laparoscopic cholecystectomy on patient outcomes. Surg Endosc 2008;22:2541-2553.

13. Thakur V, Schlachta CM, Jayaraman S. Minilaparoscopic versus conventional laparoscopic cholecystectomy a systematic review and meta-analysis. Ann Surg 2011;253: 244-258

14. Liberati A, Altman DG, Tetzlaff J, Mulrow C, Gøtzsche PC, Ioannidis JP, et al. The PRISMA statement for reporting systematic reviews and meta-analyses of studies that evaluate healthcare interventions: Explanation and elaboration. BMJ 2009;339:b2700.

15. Goossen K, Tenckhoff S, Probst P, Grummich K, Mihaljevic AL, Büchler MW, et al. Optimal literature search for systematic reviews in surgery. Langenbecks Arch Surg 2018;403:119-129.

16. Hozo SP, Djulbegovic B, Hozo I. Estimating the mean and variance from the median, range, and the size of a sample. BMC Med Res Methodol 2005;5:13.
17. Higgins JPT, Sterne JAC, Savović J, Page MJ, Hróbjartsson A, Boutron I, et al. A revised tool for assessing risk of bias in randomized trials. In: Chandler J, McKenzie J, Boutron I, Welch V. Cochrane Methods. Cochrane Database of Systematic Reviews Issue 2016;10(Suppl 1):29-31.

18. Probst P, Zaschke S, Heger P, Harnoss JC, Hüttner FJ, Mihaljevic AL, et al. Evidence-based recommendations for blinding in surgical trials. Langenbecks Arch Surg 2019; 404:273-284.

19. Ainslie WG, Catton JA, Davides D, Dexter S, Gibson J, Larvin $\mathrm{M}$, et al. Micropuncture cholecystectomy vs conventional laparoscopic cholecystectomy: A randomized controlled trial. Surg Endosc 2003;17:766-772.

20. Alponat A, Cubukçu A, Gönüllü N, Cantürk Z, Ozbay O. Is minisite cholecystectomy less traumatic? Prospective randomized study comparing minisite and conventional laparoscopic cholecystectomies. World J Surg 2002;26: 1437-1440.

21. Bisgaard T, Klarskov B, Trap R, Kehlet H, Rosenberg J. Pain after microlaparoscopic cholecystectomy. A randomized double-blind controlled study. Surg Endosc 2000;14: 340-344.

22. Bisgaard T, Klarskov B, Trap R, Kehlet H, Rosenberg J. Microlaparoscopic vs conventional laparoscopic cholecystectomy: A prospective randomized double-blind trial. Surg Endosc 2002; 16:458-464.

23. de Carvalho LF, Fierens K, Kint M. Mini-laparoscopic versus conventional laparoscopic cholecystectomy: A randomized controlled trial. J Laparoendosc Adv Surg Tech A 2013;23:109-116.

24. Planells Roig M, Arnal Bertomeu C, Garcia Espinosa R, Cervera Delgado M, Carrau Giner M. Ambulatory laparoscopic cholecystectomy by Minilaparoscopy versus traditional multiport ambulatory laparoscopic cholecystectomy. Prospective randomized trial. Cir Esp 2016;94: 86-92.

25. Saad S, Strassel V, Sauerland S. Randomized clinical trial of single-port, minilaparoscopic and conventional laparoscopic cholecystectomy. Br J Surg 2013;100:339-349.

26. Sarli L, Iusco D, Gobbi S, Porrini C, Ferro M, Roncoroni L. Randomized clinical trial of laparoscopic cholecystectomy performed with mini-instruments. Br J Surg 2003;90:13451348.

27. Schmidt J, Sparenberg C, Fraunhofer S, Zirngibl H. Sympathetic nervous system activity during laparoscopic and needlescopic cholecystectomy. Surg Endosc 2002;16: 476-480.

28. Schwenk W, Neudecker J, Mall J, Böhm B, Müller JM. Prospective randomized blinded trial of pulmonary function, pain, and cosmetic results after laparoscopic vs. microlaparoscopic cholecystectomy. Surg Endosc 2000;14: 345-348.

29. Cheah WK, Lenzi JE, So JB, Kum CK, Goh PM. Randomized trial of needlescopic versus laparoscopic cholecystectomy. Br J Surg 2001;88:45-47.

30. Huang MT, Wang W, Wei PL, Chen RJ, Lee WJ. Minilaparoscopic and laparoscopic cholecystectomy: A comparative study. Arch Surg 2003;138:1017-1023.

31. Look M, Chew SP, Tan YC, Liew SE, Cheong DM, Tan JC, et al. Post-operative pain in needlescopic versus conventional laparoscopic cholecystectomy: A prospective randomised trial. J R Coll Surg Edinb 2001;46:138142. 
32. Alhashemi M, Almahroos M, Fiore JF Jr, Kaneva $\mathrm{P}$, Gutierrez JM, Neville A, et al. Impact of miniport laparoscopic cholecystectomy versus standard port laparoscopic cholecystectomy on recovery of physical activity: A randomized trial. Surg Endosc 2017;31:2299-2309.

33. Novitsky YW, Kercher KW, Czerniach DR, Kaban GK, Khera S, Gallagher-Dorval KA, et al. Advantages of minilaparoscopic vs conventional laparoscopic cholecystectomy: Results of a prospective randomized trial. Arch Surg 2005; 140:1178-1183.

34. Di Bartolomeo N, Mascioli F, Ciampaglia F. Minilaparoscopic cholecystectomy a one year record. Ann Ital Chir 2015;86:528-531.

35. Agresta F, Bedin N. Is there still any role for minilaparoscopic-cholecystectomy? A general surgeons' last five years experience over 932 cases. Updates Surg 2012;64:31-36.

Address correspondence to: Diego Coletta, MD

Emergency Department-Emergency and Trauma Surgery Unit Umberto I University Hospital Sapienza University of Rome Viale del Policlinico 155 Rome 00161 Italy

E-mail: diegocoletta1@gmail.com 